\title{
Energy and Congestion Aware Multipath Routing in MANET
}

\author{
Mani Bushan Dsouza, Manjaiha D. H.
}

\begin{abstract}
An interconnection of wireless nodes in motion is called as Mobile AdHoc Network (MANET). One of the problems facing MANET is the route failure due to dynamic movement of nodes. Route failure leads to frequent path search and extra effort for maintaining existing path. An effective routing protocol should choose an elite path for dispatching data and consume less resources. Ad-hoc On-demand Multipath Distance Vector (AOMDV) protocol can sustain more than one path between the communicating nodes and switches between them, whenever communication fails over selected path. This way, it reduces the effort of discovering new path, whenever an existing path fails. However, while choosing alternative paths the protocol only considers the hop count as a deciding factor and it does not take into consideration the energy associated with node nor the congestion along the chosen path. In this paper, we consider both residual energy and active load while selecting path for communication. Performance of both protocols are tested on NS2 simulator. It was found that, the enhancement does provide an improvement in performance than the existing protocol.
\end{abstract}

Keywords :AOMDV, Congestion, Energy, Cost function, ECAOMDV, Power Factor, Residual Energy, Routing, MANET.

\section{INTRODUCTION}

\section{Aself-organizedmobilenodethatcommunicate}

wirelesslyin an infrastructure-less environment forms a mobile ad-hoc network [19]. Such a networks are temporary as the communication between any neighboring nodes breaks, whenever they go beyond wireless range. When a link fails between any nodes fails, all the packets that are travelling along the path are dropped, resulting in the reduction of packets delivered to the destination. This leads to a surge in end-to-end delay [1]. If there are multiple paths between the nodes, they can quickly change over to other paths, when existing path fails and still continue the communication without opting to discover a fresh route. Ad hoc On-demand Multipath Distance Vector (AOMDV) protocol uses this technique to increase its performance. It maintains multiple link-disjoint path between the communicating nodes. However, it considers only hop count while deciding on the communication path and select a path with least hop as the primary path. Energy a node and congestion level are also important factors while choosing the primary path for communication. Choosing a node with lower power can lead to early link failure due to power loss at intermediate nodes. Similar, choosing congested path can reduce the throughput of the network.

Revised Manuscript Received on January 30, 2020.

Mani BushanDszoua*, Computer Science Department,Mangalore University, Mangaluru, India. Email: mani_bushan@hotmail.com

Manjaiha D.H., Computer Science Department, Mangalore University, Mangaluru, India. Email: drmdh2014@gmail.com

\section{RELATED WORK}

There were many attempts made to improve the energy conservationand congestion control in MANET. Amultipathprotocolbased on the maximum and minimumvalue of residual energy [2] was proposed by Yumei Liu et al. This protocol advocates use of least residual energy. It uses path with highest residual energy path for data transmission. An improvement to AOMDV based on the maximizationof network lifetime was proposed by Liu et al. [3]. Here multiple paths are selected based on an energy cost function. The protocol optimizes forwarding mechanism by using multiple paths to balance energy during datatransmission.Another modification ofAOMDV based on energyconstraintwas proposed by May Cho Aye et al [4]. Here route discovery procedure is identical to AOMDV, however the node transmission power is used in determining best route. A Modification to AODV protocol to handle energy and adaptability was proposed by Patil Annapurna et al. [5]. This protocol computes drain count, based on the residual energy of the nodes and uses the least drain count paths for routing packets. Such a consideration makes the networkadaptive in nature. However, such a modification leads to higher control packetsand results obtained from simulationillustrate a better throughput for AODV than the modified protocol. SalwaOthmen et al [6] proposed a protocol based on the power and delay. This protocol takes into consideration, both life time and number of hops while routing the packets. It distributes the paths among the nodes with undue battery power. It also switches to backup path when existing path fails. The protocol executes better than SPR and MAODV, with respect to throughput, delay, and packet loss rate. One more AOMDV based protocol that considers energy while routing was proposed by Koffka Khan et al. [7]. Here the state of each node is determined by the hop count value and energy metric. Here the weakest node determines the reliability of the path and such a node is avoided during data transmission. Authors Sivaraman and Karthikeyan [8], proposed an EE-BWA-AOMDV. This protocol uses minimal energy and available bandwidth to decide the best paths. It uses the path with highest available bandwidth to transfer the data Even though this protocol shows less energy consumption, lower packet drops and an increase in delivery of packets, it may rapidly exhaust battery, as it relies on nodes with minimum energy. An enhancement to AODV protocol was proposed by Kumaran Ragunathan and ThabotharanKathiravelu [9]. Here both hop-count and packet travel time were used for choosing path during route discovery phase. 
With this enhancement, the authors are able to reduce the end to end delay and increase the packetdelivery ratio, incomparisonto AODV.Anenergy efficient optimized AOMDV using Particle Swarm Optimization (PSO) based optimization function was proposed by Aqeel et al. [10]. Here the optimizationfunction selects route having highest energy level and least distance. Simulation results of this protocol exhibits an enhancement in performance for delivery rate, end delay, and throughput in comparison with the original protocol. Alhamali MasoudAlfrgani et al [11] estimatedcongestion window and bandwidth using scheme basedoncongestioncontrol. A thorough comparison of these scheme on various window size and bandwidth are also providedby the authors. Anju et al [12] provided a congestiondetection and prevention technique based onAODV.

\section{COST FUNCTION AND ROUTE SELECTION}

Energy consumed by a Network Interface Card (NIC) with 2 Mbps and using $0.5 \mathrm{~V}$ energy, is $240 \mathrm{~mA}$ while receivingand $280 \mathrm{~mA}$ while transmitting [13]. The objective function should be designed in such a way that, it gives higher weight or cost for nodes having lower energy [14]. This way, we can eliminate lower energy nodes during route selection and obtaina stable sustainable route with nodes having higher energy.

Consider a node with residual energy $e_{i t a n d} f_{i}\left(e_{i t}\right)$ be the corresponding cost function at time $t$. This cost function hasan inverse relationship with residual energy of the node [15] and can be written as,

$$
\begin{gathered}
f_{i}\left(e_{i t}\right) \propto \frac{1}{e_{i t}} \\
f_{i}\left(e_{i t}\right)=\rho_{i} \times\left(\frac{F_{i}}{e_{i t}}\right) \times W_{i}
\end{gathered}
$$

Here, $\rho_{i}$ represents the power required by the node $n_{i}$ to transmit, $F_{i}$ is the maximum charge capacity of node $n_{i}, W_{i}$ is the weight factor whose value is based on the quality of the battery. For any intermediate node, maximum cost on a given path $\mathrm{P}_{\mathrm{j}}$ is given by,

$$
\mathrm{C}_{1}\left(\mathrm{P}_{\mathrm{j}}\right)=\max \left\{\mathrm{fi}\left(\mathrm{e}_{\mathrm{it}}\right)\right\} \forall \mathrm{ni} \in \mathrm{P}_{\mathrm{j}}
$$

For all intermediate nodes along the path $\mathrm{P}_{\mathrm{j}}$, the total cost can be represented as,

$$
C_{2}\left(P_{j}\right)=\sum_{i=1}^{n} f_{i}\left(e_{i t}\right)
$$

If there are a set of $\mathrm{M}$ multipath which are node disjoint, then feasible path at any given time $t$, is given by,

$$
\mathrm{Pf}=\min (\mathrm{C} 1(\mathrm{Pj})) \quad \forall \mathrm{Pj} \in \mathrm{M}
$$

In other words, a path having an intermediate node with least maximum cost can be considered as feasible path. Suppose, if the source and destination have F such feasible paths, then optimal path among them, is the one having least total cost among all the nodes within a path. This is given as,

$$
\mathrm{P}_{\mathrm{o}}=\min (\mathrm{C} 2(\mathrm{Pj})) \forall \mathrm{Pj} \in \mathrm{F}
$$

Congestion of a link connecting two nodes can estimated by considering the size of the buffer [16]. To estimate the congestion along the path, occupied buffer size of all nodes along the path are added. This value can be used to choose the optimal paths from the available alternative paths. [10]. Congestion Level for given path can be calculated using equation (7).

$$
\text { Congestion Level }=\frac{1}{n_{p}} \sum_{i=1}^{n_{p}} \text { buffer_size }(i)
$$

Here $\mathrm{n}_{\mathrm{p}}$ indicates the number of hops in the route $p$. The occupied buffer size for the link $i$ of the route $p$ is given by buffer_size $(i)$.

\section{WORKING OF MODIFIED AOMDV PROTOCOL}

AOMDV creates a link disjoint, multiple paths among communicatingnodes. AOMDV differentiate the paths basedon the number of hops and chooses a path with minimum number of hops for communication.It tries to selecta pathwith leastnumber of hops.However, consideringonly number of hops does not always gives optimal results and we must also consider the cost and congestion during the path selection [17]. The Energy and Congestion Ad-hoc On-demandMultipath Distance Vector (ECAOMDV) protocoltakesinto account both cost and congestion along the path. The RREQ packet of ECAOMDV is enhanced by adding following fields shown in Fig 1.

\begin{tabular}{|c|c|c|c|}
\hline $\begin{array}{c}\text { Hop } \\
\text { Count }\end{array}$ & $\begin{array}{c}\text { Buffer } \\
\text { Size }\end{array}$ & Cost & $\begin{array}{c}\text { Max } \\
\text { Cost }\end{array}$ \\
\hline
\end{tabular}

Fig. 1.Modified Route Request packet of ECAOMDV

Where Hop-Count is the count of hops amid the source and current node. Similarly, changes in RREP structure is

\begin{tabular}{|c|c|c|c|c|c|c|c|c|c|}
\hline $\begin{array}{l}\text { Destiantion } \\
\text { Address }\end{array}$ & Seq..$N_{0}$ & $\begin{array}{l}\text { Advertise } \\
\text { hopcount }\end{array}$ & & & & & & $\begin{array}{l}\text { Max } \\
\text { Cost }\end{array}$ & cost \\
\hline & & & $\begin{array}{l}\text { Next- } \\
\text { hop1 }\end{array}$ & $\begin{array}{l}\text { Last- } \\
\text { hop1 }\end{array}$ & $\begin{array}{l}\text { Hop } \\
\text { count1 }\end{array}$ & Timeout1 & $\overline{\text { CL1 }}$ & & \\
\hline & & & $\begin{array}{l}\text { Nert- } \\
\text { hopp } 2\end{array}$ & $\begin{array}{l}\text { Last- } \\
\text { hopp }\end{array}$ & $\begin{array}{l}\text { Hop_ } \\
\text { count2 }\end{array}$ & Timeout2 & $\mathrm{CL2}$ & & \\
\hline
\end{tabular}
revealed in Fig 2.

\begin{tabular}{|c|c|c|c|}
\hline $\begin{array}{c}\text { Hop } \\
\text { Count }\end{array}$ & $\begin{array}{c}\text { Buffer } \\
\text { Size }\end{array}$ & Cost & $\begin{array}{c}\text { Max } \\
\text { Cost }\end{array}$ \\
\hline
\end{tabular}

Fig. 2.Enhanced Route Reply packet of ECAOMDV

Changes to the routing table includes, adding additional fields such as Residual Energy (RE), Max Cost, Congestion Level (CL) and Cost. This is shown in Fig 3.

Fig. 3.Modified Routing Table of ECAOMDV

Whenever a source-node needs to transmit data to another node, whose path is not yet fabricated, it broadcasts RREQ packets its neighbors. When such a RREQ packet is received by intermediate node, it starts a timer and stores the Cost value of RREQ field.Any subsequent route requestsfrom the same source, which has taken different path is broadcasted only if they meet following conditions. 
IF RREQ.Cost<Node.Cost THEN

Broadcast RREQ

ELSE IF RREQ.Cost $=$ Node. Cost THEN

IF RREQ.max_cost<Node.max_cost THEN

Broadcast RREQ

ELSE IF RREQ.max_cost $=$ Node.max_cost THEN

IF RREQ.hopCount<Node.Advertised_hopCountTHEN

Broadcast RREQ

END IF

END IF

ENF IF

Before broadcasting RREQ, the intermediate node performs following tasks.

1) Increment RREQ.HopCount by one.

2) If Node.max_cost is greater than RREQ.Max_Cost, then RREQ.Max_Cost $=$ Node.max_cost.

3) RREQ.Cost $=$ RREQ.Cost $+\operatorname{Node}($ Cost $)$ that is add the value of cost funciton

4) If Node.cost is greater than RREQ.Cost, then RREQ.Cost $=$ Node.cost.

5) Computer RREQ. buffer_size = buffer_size + occupied size of the buffer of link

6) Update RoutingTable.CL = buffer_size / hopcount

When RREQ is received by the destination, it computes the total cost of the path and sends a RREP through each node disjoint path. The RREP contains the cost, Max Cost and Buffer Size which can be used to by the source for data transfer. From the multiple paths, available for the source, a path with least congestion is used for transferring data packets. When an existing path breaks between any nodes, a RERR packet is generated and the route maintenance process gets initiated.

\section{PERFORMANCE ANALYSIS}

The NS-2.34 simulation framework is used to compare the performances of AOMDV and ECAOMDV. Simulation environmentconsists of a 1000 by 1000 square meter flat area with 40 moving wireless nodes. Random way-point mobility model is used during simulation [20]. Various parameters such as Average Delay, Traffic Overhead and Packet Delivery ratio are measured by varying number of connections. Details of simulation is shown in Table I.

\section{A. Packet Delivery Ratio}

Generally, protocols that show higher values of PDR are considered to be better. It was observed that with the packet delivery ration is slightly better than AOMDV. This is because, ECAOMDV selects less congested path for transmission. As the path has less congestion, there are less chances of packets being dropped and this increases PDR. Also, ECAOMDV chooses nodes with sufficientpower. Thisalso prevents link breakage due to node failure. Finally, when the path breaks, RERR is used to restore the path. All these factors lead to a higher value of PDR as plotted in Fig 4.

\section{B. Routing Overhead}

It was observed that the routing overhead increases with time, but ECOMDV shows a lower trend in routing overhead. This may be due to the fact that the path selected by EAOMDV are stable and does not need to be re-established due to path failure. Thus, reducing the control packets. This is obvious from the Fig 5.

Table-I: Settings of simulation

\begin{tabular}{|l|l|}
\hline \multicolumn{1}{|c|}{ Parameter } & \multicolumn{1}{c|}{ Value } \\
\hline Dimensions & 0.7 Joule / packet \\
\hline Transmission Power & 0.3 Joule / packet \\
\hline Receiving Power & 100 Joules \\
\hline Initial Energy & 100 Joules \\
\hline Maximum Battery Capacity & 10 \\
\hline Weight factors & 40 \\
\hline Number of Nodes & 30 \\
\hline Number of Connections & CBR \\
\hline Source Type & 512 bytes \\
\hline Packet Size & Drop tail \\
\hline Queue Type & $50 * 512$ bytes \\
\hline Queue Size & 802.11 b \\
\hline Mac Layer & Random Way Point \\
\hline Simulation Model & AOMDV, ECAOMDV \\
\hline Routing Protocols & \\
\hline
\end{tabular}

\section{Variation of Packet Delivery Ratio with TIme}

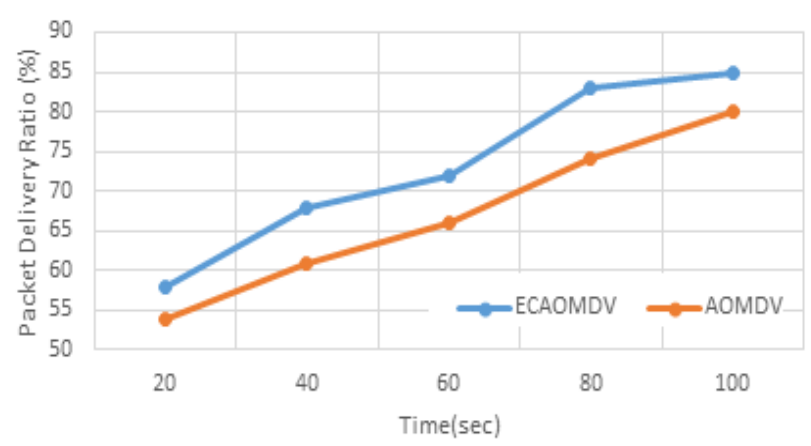

Fig. 4.Increase of $\%$ of PDR with time Variation of Routing Overhead with Time

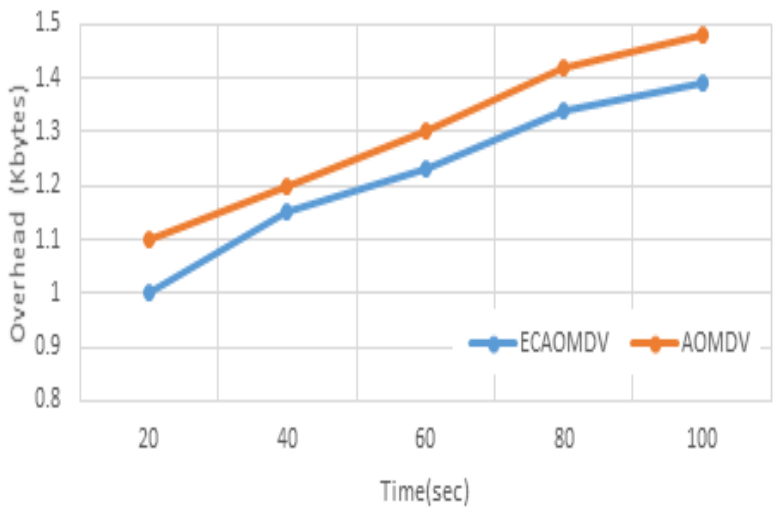

Fig. 5.Variation of routing overhead with time

\section{Average end-to-end delay}

The Fig 6, shows that the movement of nodes increases with time and it leads to the breakage of link. 
This contributes to an increase in delay. However, it can be seen that the delay is less in ECAOMDV during initial stage and the delay converges with AOMDV at later time.

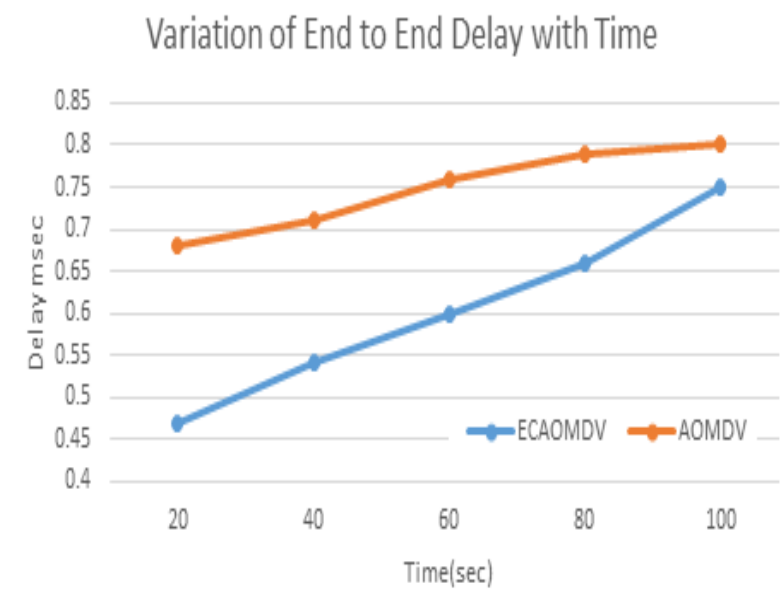

Fig. 6.Variation of End to End Delay with time

\section{Residual Energy}

Simulation shows that ECAOMDV utilizes lower energy in comparison with AOMDV. This is because, ECAOMDV avoids nodes with lower residual energy and distributes the load fairly across all nodes. As a result, we observe that, the average residual power of all nodes decreases steadily in comparison with AOMDV. This is evident in Fig 7.

\section{Variation of Energy with Time}

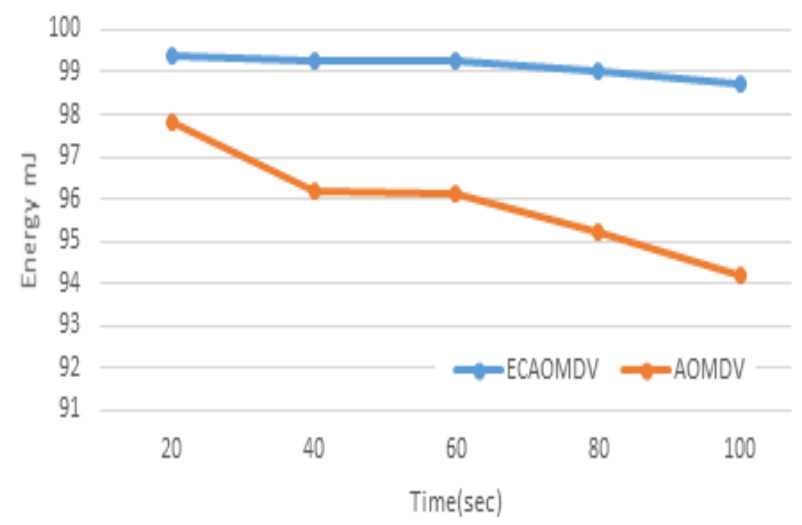

Fig. 7.Decrease in Residual Energy with time

\section{E. Throughput}

Simulation results shows that, throughput of ECAOMDV increases with time. This is due selection of less congested and stable path. Throughput comparison is plotted in Fig8.

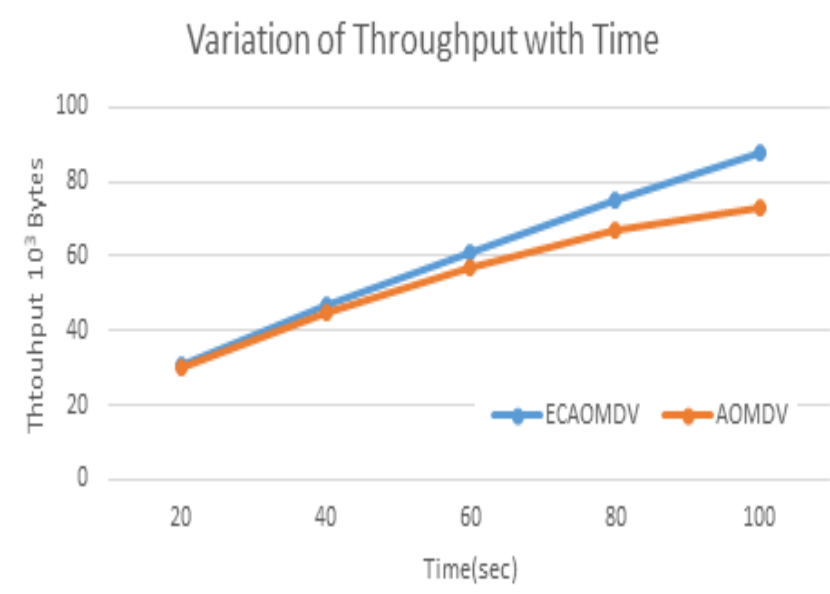

Fig. 8.Increase in Throughput with time

\section{CONCLUSIONS}

The proposed, Energy and Congestion ware protocol selects an optimal path subject to the level of congestion and residual power of the nodes. It performs a fair allocation of load between the nodes and improves lifetime of the route, thereby providing better throughput for the network. Result of simulation shows that, ECAOMDV consumes less energy and delivers more packets as compared to AOMDV.

\section{REFERENCES}

1. V. G. Jayshree Tajne, "MultipathNode-DisjointRoutingProtocol to Minimize End to End Delay and Routing Overhead for Manets," International Journal of Engineering Research and Applications, vol. 3 , no. 4, August 2013.

2. Yumei Liu, Lili Guo, Huizhu Ma and Tao Jiang, "Energy efficient on demand multipath routing protocol for multihop ad hoc networ ks,"

ISSSTA-08, IEEE 10th InternationalsymposiumonSpreadspectrum a nd applications, Bologna, Italy, August 2008.

3. Juanwei Liu, Jian Chen and YonghongKuo, "Multipath Routing Protocol for Networks Lifetime Maximization in Ad-Hoc Networks. Proc. of the 5th International Conference on Wireless Communications"Networking and Mobile Computing (WiCom '09), pp 1-4, 2019.

4. May Cho Aye and Aye Moe Aung,"A Modified Energy Constrained Protocol based on AOMDV for MobileAd-HocNetworks," International Journal of Scientific Engineering and Technology Research, vol. 3, November 2014.

5. A. P. Patil, B. V. Chandan, S. Aparna and R. Greeshma, "An improved energyefficient AODV routing protocol for MANETs," Eleventh International Conference on Wireless and Optical Communications Networks (WOCN), pp. 1-5, 2014.

6. SalwaOthmen, AymenBelghith, FaouziZarai and Mohammad S. Obaidat,"Power and Delay-aware Multi-path Routing Protocol for Ad Hoc Networks," 2014 International Conference on Computer, Information and Telecommunication Systems (CITS), Jeju, pp. 1-6, 2014 .

7. Koffka Khan and Wayne Goodridge, "Energy Aware Ad Hoc On-Demand Multipath Distance Vector Routing," International Journal of Intelligent Systems and Applications (IJISA),2015.

8. Sivaraman T. and Karthikeyan E., "EE-BWA-AOMDV: Energy Efficientand Bandwidth Aware On-demand Multipath Routing protoco for Mobile Ad hoc Networks," International Journal of Computer Application, vol. 6, no. 2, pp. 85-99, 2016.

9. K. Ragunathan and T. Kathiravelu, "A hop-count and time-based MANETrouting protocol," 2016 IEEE International Conference on Advanced Networks and Telecommunications Systems (ANTS), pp. $1-6,2016$. 
10. A. Taha, R. Alsaqour, M. Uddin, M. Abdelhaq and T. Saba, "Energy Efficient Multipath Routing Protocol for Mobile Ad-Hoc Network Using the Fitness Function," IEEE Access, vol. 5, pp. 10369-10381, 2017.

11. AlhamaliMasoudAlfrgani Ali, RaghavYadav and HariMohan Singh, "Congestion Control Technique for Wireless Networks," IOSR Journal of Computer Engineering(IOSR-JCE), vol. 16, no. 2, Ver. II, pp. 31-33, Mar-Apr. 2014.

12. Anju and SugandhaSingh, "Modified AODV for Congestion Control in MANET," International Journal of Computer Science and Mobile Computing, vol. 4, no. 6, pp. 984-1001, June-2015.

13. Juan-Carlos C and Dongkyun Kim, "Investigating Performance of Power-aware Routing Protocols for Mobile Adhoc Networks," in International Mobility and Wireless Access Workshop, Fort Worth, TX, USA, 2002.

14. Diwan B. and Sumalatha M.R., "Transmission and Reception Based Relay Deployment for Database Transactions in MANETs," in Advancesin Computing,Communication and Control. ICAC32013. Communications in Computer and Information Science, vol 361., Berlin, Heidelberg, Springer, 2013.

15. M Bheemalingaiah, MM. Naidu, D. Sreenivasa Rao and P.Vishva pathi,"Energy Aware On-Demand Multipath Routing Protocolin Mobile Ad Hoc Networks," IRACST - International Journal of Computer Networks and Wireless Communications (IJCNWC), vol. 6, no. 5, pp. 14-31, 2016.

16. M. Tekaya, N. Tabbane and S. Tabbane, "Multipath routing mechanism with load balancing in ad hoc network," in The 2010 International Conference onComputer Engineering \& Systems, Cairo, pp. 67-72, 2010.

17. M.K.Marina and S.R.Das, "On-Demand multipath distance vector routing in ad hoc networks," in 9th IEEEInternationalConference on Network Protocols (ICNP), Riverside, CA, USA, 2001,pp.14-23.

18. Y. HaroldRobinson, E. Golden Julie, KrishnanSaravanan, RaghvendraKumar and Le Hoang Son, "FD-AOMDV: fault-tolerantdisjointad-hocon-demand multipath dista nce vector routingalgorithmin mobile ad-hoc networks," Journal of Ambient Intelligence and Humanized Computing, Springer Berlin Heidelberg, pp. 1-18, 2018.

19. Dipika Sarkar, SwagataChoudhury, AbhishekMajumder "Enhanced-Ant-AODVfor optimal route selection in mobile ad-hoc network",Journalof King Saud University - Computer and Information Sciences, 2018.

20. S. M. Benakappa, M. Kiran. "An energy-aware node disjoint multipath routing protocol for MANETs with dynamictransmission range adjustment",2017 International Conference on Electrical, Electronics, Communication, Computer, and Optimization Techniques(ICEECCOT), 2017.

\section{AUTHORS PROFILE}

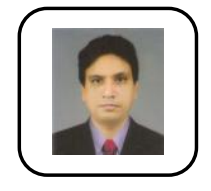

Mr. Mani Bushan Dsouza, is a research scholar at Mangalore University, located in Dakshina Kannada District of Karnataka State in India. He is currently working on enhancing routing in Mobile Ad hoc networks. He has published papers in UGC recognized journals.

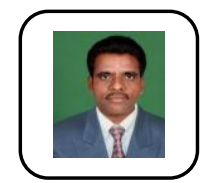

Dr. Manjaiah D.H, is chairperson and professor at Computer Science Department in Manglore university. He has published over 50 papers in International and National Journals Publications.He has over 50 Publications International and National Conference Proceedings 